\title{
Ferromagnetic-Insulator-Based Superconducting Junctions as Sensitive Electron Thermometers
}

\author{
F. Giazotto, ${ }^{1, *}$ P. Solinas, ${ }^{2}$ A. Braggio, ${ }^{2,3}$ and F. S. Bergeret ${ }^{4,5, \uparrow}$ \\ ${ }^{1}$ NEST Istituto Nanoscienze-CNR and Scuola Normale Superiore, I-56127 Pisa, Italy \\ ${ }^{2}$ SPIN-CNR, Via Dodecaneso 33, 16146 Genova, Italy \\ ${ }^{3}$ I.N.F.N. Sezione di Genova Via Dodecaneso 33, 16146, Genova, Italy \\ ${ }^{4}$ Centro de Física de Materiales (CFM-MPC), Centro Mixto CSIC-UPV/EHU, \\ Manuel de Lardizabal 4, E-20018 San Sebastián, Spain \\ ${ }^{5}$ Donostia International Physics Center (DIPC), Manuel de Lardizabal 5, E-20018 San Sebastián, Spain \\ (Received 4 June 2015; revised manuscript received 14 July 2015; published 26 October 2015)

\begin{abstract}
We present an exhaustive theoretical analysis of charge and thermoelectric transport in a normal-metalferromagnetic-insulator-superconductor junction and explore the possibility of its use as a sensitive thermometer. We investigate the transfer functions and the intrinsic noise performance for different measurement configurations. A common feature of all configurations is that the best temperature-noise performance is obtained in the nonlinear temperature regime for a structure based on an Europium chalcogenide ferromagnetic insulator in contact with a superconducting $\mathrm{Al}$ film structure. For an opencircuit configuration, although the maximal intrinsic temperature sensitivity can achieve $10 \mathrm{nK} \mathrm{Hz}^{-1 / 2}$, a realistic amplifying chain will reduce the sensitivity up to $10 \mu \mathrm{K} \mathrm{Hz}^{-1 / 2}$. To overcome this limitation, we propose a measurement scheme in a closed-circuit configuration based on state-of-the-art superconductingquantum-interference-device detection technology in an inductive setup. In such a case, we show that temperature-noise can be as low as $35 \mathrm{nK} \mathrm{Hz}^{-1 / 2}$. We also discuss a temperature-to-frequency converter where the obtained thermovoltage developed over a Josephson junction operated in the dissipative regime is converted into a high-frequency signal. We predict that the structure can generate frequencies up to approximately $120 \mathrm{GHz}$ and transfer functions up to $200 \mathrm{GHz} / \mathrm{K}$ at around $1 \mathrm{~K}$. If operated as an electron thermometer, the device may provide temperature-noise lower than $35 \mathrm{nK} \mathrm{Hz}^{-1 / 2}$ thereby being potentially attractive for radiation-sensing applications.
\end{abstract}

DOI: 10.1103/PhysRevApplied.4.044016

\section{INTRODUCTION}

Recent theories have shown that the spin-splitting induced in a superconductor $(S)$ placed in contact with a ferromagnetic insulator (FI) can be exploited in different kinds of spin caloritronic devices such as heat valves [1,2] or thermoelectric elements [3-6]. They can be used as building blocks in phase-coherent thermoelectric transistors [7] and for the creation of magnetic fields induced by a temperature gradient in Josephson junctions (JJs) due to the thermophase effect [8]. Normal-metal-ferromagneticinsulator-superconductor $(N-\mathrm{FI}-S)$ junctions have been also proposed for efficient electron cooling [9] of the normal metal $N$ [10]. The possible applications of superconductor-ferromagnetic structures for thermoelectrics has been also highlighted in a recent review article [11].

In the present work, we theoretically analyze charge and thermoelectric transport in a prototype structure based on the FI- $S$ building block and explore its application as an ultrasensitive electron thermometer [12-19] and eventually as a temperature-to-frequency converter. Our system consists of a $\mathrm{N}$-FI-S junction denoted here as the TE, which is

\footnotetext{
*francesco.giazotto@sns.it

†sebastian_bergeret@ehu.es
}

connected via the superconducting wires $S_{1}$ to a generic load element, as shown in Fig. 1. A temperature difference localized between the $N$ and $S$ sides of the TE induces a thermoelectric signal [4]. We consider three different configurations of the load resistance $R_{L}=\infty$ (open circuit), $R_{L}=0$ (closed circuit), and finite load $R_{L}=R_{\mathrm{JJ}}$ where we close the system over a generic Josephson element in the dissipative regime with shunting resistance $R_{\mathrm{JJ}}$. Depending on the configuration, the device will operate in different regimes: (i) Seebeck regime, where a Seebeck thermovoltage $(V)$ is generated across the TE element at open-circuit, and (ii) Peltier regime, where the gradient of the temperature generates a circulating thermocurrent that can be probed by an inductive measurement for closed circuit. Here we explore both regimes, which includes an estimate of the intrinsic noise and the best expected temperature sensitivity with state-of-the-art technology for signal detection. We discuss the advantages and drawbacks of the different configurations and show that operated within the nonlinear regime, the intrinsic noise of the device is reduced. In particular, our numerical results show that the noise performance is mainly determined by the junction differential resistance $R_{d}$, which can be drastically reduced beyond the linear-response regime with respect to the temperature. We finally discuss how the 


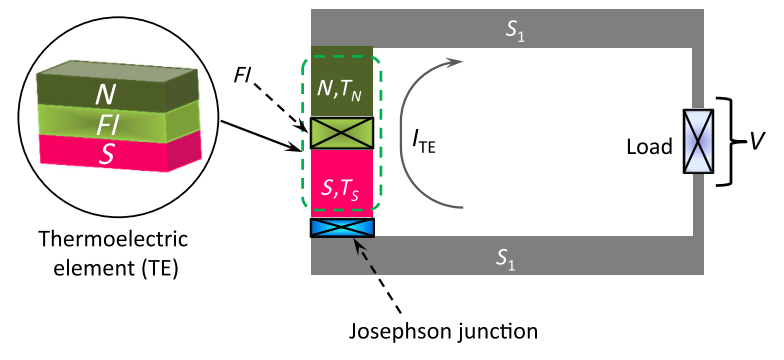

FIG. 1. General scheme of the device based on a $N$-FI-S junction shown in the enlargement as stacked layers of different materials. $T_{S}$ and $T_{N}$ denote the temperature in $S$ and $N$, respectively, $I_{\mathrm{TE}}$ is the thermocurrent circulating in the circuit, and $V$ is the thermovoltage developed across the TE. $S_{1}$ is a superconductor contacted to both ends of the TE which contains a load with resistance $R_{L}$. The load is intended to be an open-circuit $R_{L} \rightarrow \infty$, a dissipationless closed-circuit $R_{L}=0$, or in the form of a generic Josephson element operated in the dissipative regime in order to convert the thermovoltage $V$ into radiation at the Josephson frequency.

generated thermovoltage can induce an ac-Josephson effect with a supercurrent oscillating at a frequency $\nu=|V| / \Phi_{0}$ [20], where $\Phi_{0} \simeq 2.067 \times 10^{-15} \mathrm{~Wb}$ is the flux quantum. The frequency $\nu$ can be measured with great accuracy providing accurate and fast information about temperature difference across the TE.

The paper is organized as follows: In Sec. II we briefly present the general formalism and the expressions for the electric current flowing through the $N$-FI- $S$ junction and the noise as a function of all the parameters involved in the system. With the help of this expression, we analyze in Sec. II A the electric and thermoelectric response of the TE in the nonlinear-response regime. In particular, we show the impact of the exchange field as well as the role of the barrier polarization on the charge current. In Sec. II B we discuss the different measurement configurations of the device analyzing the effect of the load resistance $R_{L}$ over the thermoelectrical properties of TE recalling the results for the linear regime in Sec. IIC. The evaluation of the intrinsic noise properties of the $N$-FI-S junction is done both for the linear and nonlinear regimes. Assuming a realistic device based on europium sulfide (EuS) as a FI and superconducting aluminum (Al) operating at low temperatures, we discuss the open-circuit and closed-circuit configurations, respectively, in Secs. III and IV. In those sections, we also discuss the temperature-noise performance taking into account the most simple measurement scheme with actual state-of-the-art technologies. Finally, in Sec. V, we discuss the temperatureto-frequency conversion scheme where the thermovoltage developed across the $N$-FI-S junction is converted into a high-frequency signal by a Josephson element driven into the dissipative regime. The full temperature-to-frequency conversion capability of the $N$-FI- $S$ junction is analyzed, investigating as well the temperature-noise performance. We summarize our results in Sec. VI.

\section{MODEL}

It is instructive to start with the description of the $N$-FI-S building block. The interaction between the spin of the conducting electrons in $S$ and the localized magnetic moments in a FI lead to an effective exchange interaction in $S$ that decays over the superconducting coherence length $\xi_{0}$ [21]. We assume that the $S$ layer is thinner than $\xi_{0}$, so that the exchange field $\left(h_{\mathrm{exc}}\right)$ induced in $S$ by the FI is spatially homogenous. In such a case, the superconductor density of the states (DOS) is given by the sum of the densities for spin-up $(\uparrow)$ and spin-down $(\downarrow)$ quasiparticles,

$$
N_{\uparrow, \downarrow}(E)=\frac{1}{2}\left|\operatorname{Re}\left[\frac{E+i \Gamma \pm h_{\mathrm{exc}}}{\sqrt{\left(E+i \Gamma \pm h_{\mathrm{exc}}\right)^{2}-\Delta^{2}}}\right]\right| .
$$

Here, $\Delta\left(T_{S}, h_{\mathrm{exc}}\right)$ is the pairing potential that depends both on temperature $T_{S}$ in $S$ and $h_{\text {exc }}$, and it is computed selfconsistently in a standard way [22] from the gap equation

$$
\ln \left(\frac{\Delta_{0}}{\Delta}\right)=\int_{0}^{\hbar \omega_{D}} d E \frac{f_{+}(E)+f_{-}(E)}{\sqrt{E^{2}+\Delta^{2}}},
$$

where $f_{ \pm}(E)=\left\{1+\exp \left[\left(\sqrt{E^{2}+\Delta^{2}} \mp h_{\mathrm{exc}}\right) / k_{B} T_{S}\right]\right\}^{-1}$, $\omega_{D}$ is the Debye frequency of the superconductor, $\Delta_{0}$ is the zero-temperature and zero-exchange field superconducting pairing potential, and $k_{B}$ is the Boltzmann constant. Furthermore, $\Gamma$ accounts for broadening, and for an ideal superconductor $\Gamma \rightarrow 0^{+}$[23].

We are interested in the current through the $N$-FI-S junction, which in the tunneling limit considered here is given by [4]

$I_{\mathrm{TE}}=\frac{1}{e R_{T}} \int_{-\infty}^{\infty} d E\left[N_{+}+P N_{-}\right]\left[f_{N}\left(V, T_{N}\right)-f_{S}\left(T_{S}\right)\right]$.

Here, $R_{T}$ is the normal-state resistance of the tunneling junction and $N_{ \pm}=\left(N_{\uparrow} \pm N_{\downarrow}\right)$. Notice that in the tunneling limit, the Andreev reflection is negligibly small, and, hence, no superconducting proximity effect in $N$ takes place. We assume thermalization on both the $S$ and $N$ layers neglecting any deviation of the distribution functions from their equilibrium form [24]: $f_{S}\left(T_{S}\right)=\left[1+\exp \left(E / k_{B} T_{S}\right)\right]^{-1}$ and $f_{N}\left(V, T_{N}\right)=\left\{1+\exp \left[(E+\mathrm{eV}) / k_{B} T_{N}\right]\right\}^{-1}$. Here, $T_{N}$ is the temperature in the $N$ layer, and $-e$ is the electron charge. The role of the FI layer is twofold: it acts as a spin filter with polarization [25] $P=\left(G_{\uparrow}-G_{\downarrow}\right) /\left(G_{\uparrow}+G_{\downarrow}\right)$ and causes the spin splitting of the DOS in the $S$ layer due to the exchange coupling between the localized magnetic moments of the FI and the conducting electrons of $S[21,26,27]$. These two features have been demonstrated in several experiments [28-33]. Notice that according to Eq. (3), even in the absence of a voltage bias across the junction, a finite current $I_{\mathrm{TE}}$ can flow provided $T_{N} \neq T_{S}$, as demonstrated in Ref. [4]. 


\section{A. Electric and thermoelectric response of the TE}

Before analyzing the role of a temperature bias across the TE, we determine the current-voltage characteristics (IVCs) and differential conductance $G=d I_{\mathrm{TE}} / d V$ of the $N$-FI- $S$ junction. We set a low temperature, $T_{N}=T_{S}=0.01 T_{c}$, where $T_{c}=\Delta_{0} /\left(1.764 k_{B}\right)$ is the critical temperature of the superconductor.

The results obtained from Eq. (3) are summarized in Fig. 2. Figures 2(a) and 2(b) show the IVC and $G$, respectively, for a polarization of the barrier $P=50 \%$ and different values of the spin-splitting exchange field $h_{\text {exc }}$. In Fig. 2(a), one clearly sees the deviation of the IVCs from those of a metal-insulator-superconductor $(N-I-S)$ junction. For finite values of $h_{\text {exc }}$, there is a sizeable subgap current [see Fig. 2(a)] as a consequence of the spin splitting of the DOS in the $S$ electrode. This splitting manifests itself also in the differential conductance $G$ [see Fig. 2(b)], where the coherent peaks, usually appearing at $V= \pm \Delta / e$, are now split in four peaks appearing at $V=\left( \pm \Delta \pm h_{\mathrm{exc}}\right) / e$. The asymmetry in the height of the coherent peaks stems from the spin polarization $P$ of the FI barrier [see Figs. 2(c) and 2(d)] where we set $h_{\mathrm{exc}}=0.4 \Delta_{0}$, and the curves are calculated for different values of $P$. Therefore, from IVCs one can estimate both the polarization of the barrier and the spin splitting induced in $S$ [28].
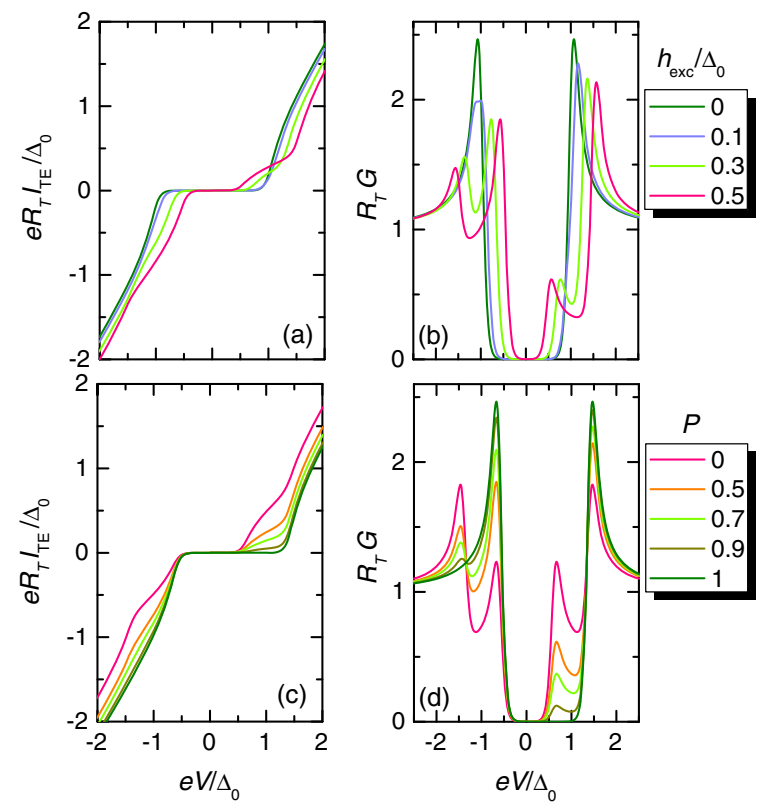

FIG. 2. Characterization of the TE for $T_{S}=T_{N}$. (a) Current vs voltage $\left(I_{\mathrm{TE}}-V\right)$ characteristics of the TE element calculated at $T_{S}=T_{N}=0.1 T_{c}, P=0.5$ and for a few values of $h_{\mathrm{exc}}$. (b) Differential conductance vs voltage $(G-V)$ characteristics of TE calculated for the same parameters as in panel (a). (c) $I_{\mathrm{TE}}-V$ and (d) $G-V$ characteristics of TE calculated at $T_{S}=T_{N}=0.1 T_{c}$, $h_{\text {exc }}=0.4 \Delta_{0}$, and for a few values of $P . \Delta_{0}=1.764 k_{B} T_{c}$ is the zero-temperature, zero-exchange-field superconducting gap, $T_{c}$ denotes the critical temperature, and $R_{T}$ is the normal-state resistance of the TE.

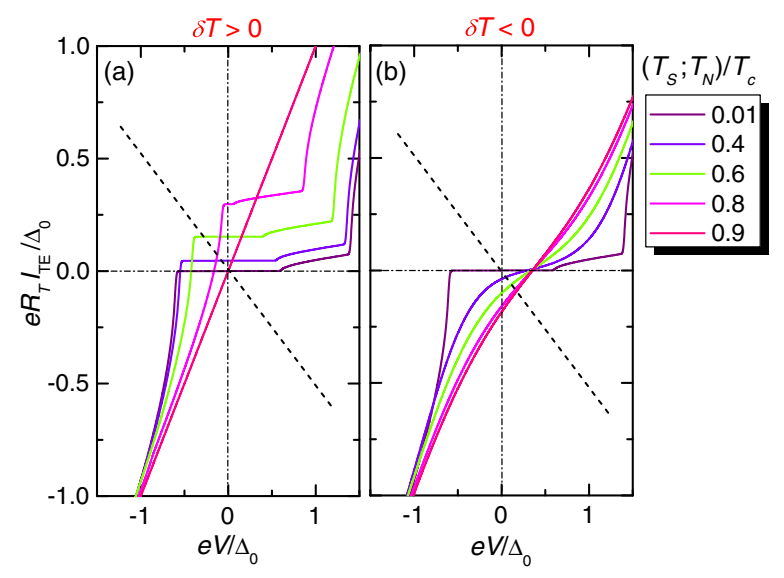

FIG. 3. Characterization of the TE for $T_{S} \neq T_{N}$. (a) $I_{\mathrm{TE}}-V$ characteristic of TE calculated for several values of $T_{S}$ (see top legend) at $T_{N}=0.01 T_{c}, P=0.9$, and $h_{\mathrm{exc}}=0.4 \Delta_{0}$. (b) The same as in panel (a) calculated for several $T_{N}$ values at $T_{S}=0.01 T_{c}$. Dashed lines in panels (a) and (b) represent the current $\left(I_{\mathrm{JJ}}\right)$ flowing through the Josephson element when it is operated in the resistive regime, $I_{\mathrm{JJ}}=-V / R_{\mathrm{JJ}}$.

We now assume a finite-temperature difference between the electrodes [34] $\delta T=T_{S}-T_{N}$ and recalculate the IVCs from Eq. (3) for $h_{\mathrm{exc}}=0.4 \Delta_{0}$ and $P=0.9$. The results are shown in Figs. 3(a) and 3(b), where we keep one of the electrodes at temperature $0.01 T_{c}$ and change the other electrode temperature. The curves in Fig. 3 reveal two main properties of the IVC. First, the IVC strongly depends on the amplitude of the temperature difference $\delta T$ : the larger the temperature difference, the larger is the current flow at low voltages. In the case that the $S$ electrode is heated [see Fig. 3(a)], this trend is limited by the reduced critical temperature $T_{c}^{*}<T_{c}$ of the superconductor originating from the presence of a finite $h_{\mathrm{exc}}$ which suppresses the $\Delta\left(T_{S}, h_{\text {exc }}\right)$ calculated self-consistently. When $T_{S} \rightarrow T_{c}^{*}$, the TE is driven into the normal state with an Ohmic characteristic [the red curve in Fig. 3(a)].

Second, there is another interesting feature of the IVCs: they strongly depend on the sign of $\delta T$. For the same value of $|\delta T|$, the current at $V=0$ is larger when the $N$ electrode is colder than the $S$ one, i.e., when $\delta T>0$. In other words, the thermoelectric effect in the TE strongly depends on the temperature difference. This feature was not investigated in previous works $[3,4]$ in which only the linear-response regime was discussed.

\section{B. Measurement configurations}

To use the TE element as a thermometer, we need to extract from the thermoelectrical signal the temperature gradient present across the TE junction. In order to do so, we need to close the TE circuit over a generic load element modeled with a load resistance $R_{L}$ (see Fig. 1). In such a case, the voltage $V$ developed across the TE for a given $\delta T$ is the solution of the following nonlinear integral equation 


$$
I_{\mathrm{TE}}\left(V, T_{S}, T_{N}, h_{\mathrm{exc}}, P\right)+\frac{V}{R_{L}}=0,
$$

where $I_{\mathrm{TE}}$ is defined in Eq. (3). The solution to the above equation is given by the point in which the dashed line (with slope proportional to $1 / R_{L}$ ) in Figs. 3(a) and 3(b) intersects the IVCs.

For a Seebeck-like measurement, one needs to maximize the thermovoltage opening the circuit, i.e., $R_{L} \rightarrow \infty$ and $I_{\mathrm{TE}}=0$. For a Peltier-like measurement, one needs to maximize the current closing the circuit with a superconducting loop, i.e., $R_{L}=0$ and, consequently, $V=0$. In the case of temperature-to-frequency conversion that we discuss later, one needs to include a Josephson element that operates in the dissipative regime with a load resistance $R_{L}=R_{\mathrm{JJ}}$, which is the total shunting resistance of the Josephson element.

Independent of the chosen configuration, we assume to connect the TE to the detector with two superconducting arms $S_{1}$. In particular, we assume to place a tunnel barrier between $S$ and $S_{1}$ to isolate the $S$ element thereby ensuring its description as a thermally homogeneous superconductor with a spin-split DOS. We neglect here any influence of $S_{1}$ arms such that the current through the TE is described by Eq. (3). Superconductors $S$ and $S_{1}$ are Josephson coupled through the barrier so that no additional voltage drop will occur. Furthermore, we also assume the $N S_{1}$ junction to be a clean metallic contact, thereby contributing negligibly to the total resistance of the system, and, for simplicity, we disregard the proximity effect induced into the $N$ layer by the nearby contacted superconductor $S_{1}$ [22].

\section{Linear-response regime}

In the linear-response regime, i.e., when the voltage $V$ and temperature difference $\delta T \ll T \equiv\left(T_{S}+T_{N}\right) / 2$ across the $N$-FI- $S$ junction are small, Eq. (4) reads $[3,4]$

$$
I_{\mathrm{TE}} \approx I_{\mathrm{TE}}^{\mathrm{lin}}=\sigma V+P \alpha \frac{\delta T}{T},
$$

where

$$
\sigma=\frac{1}{R_{T}} \int_{-\infty}^{\infty} d E \frac{N_{+}}{4 k_{B} T \cosh ^{2}\left(\frac{E}{2 k_{B} T}\right)}
$$

is the electric conductance, and $\alpha$ is the thermoelectric Seebeck coefficient [4] defined as

$$
\alpha=\frac{1}{e R_{T}} \int_{-\infty}^{\infty} d E \frac{E N_{-}}{4 k_{B} T \cosh ^{2}\left(\frac{E}{2 k_{B} T}\right)},
$$

which in the linear regime is connected to the Peltier coefficient $\Pi=\alpha T$ by Onsager symmetry. Substituting Eq. (5) in Eq. (4) and solving with respect to the thermovoltage across the TE element, one finds

$$
V^{\mathrm{lin}} \simeq-P \alpha \frac{R_{L}}{R_{L} \sigma+1} \frac{\delta T}{T}
$$

which is valid in the linear-response regime assuming a generic load resistance $R_{L}$. We see immediately that the thermovoltage directly measures the temperature gradient in the TE. Furthermore, for fixed load resistance, the achievable thermovoltage $V$ increases with the polarization $P$. In an open-circuit configuration $\left(R_{L} \rightarrow \infty\right.$ and $\left.I_{\mathrm{TE}}=0\right)$, the TE thermovoltage is maximal being

$$
V^{\operatorname{lin}} \simeq-\frac{P \alpha}{\sigma} \frac{\delta T}{T} .
$$

For the closed circuit $\left(R_{L}=0\right.$ and $\left.V=0\right)$ instead, the thermocurrent is maximal being

$$
I_{\mathrm{TE}}^{\mathrm{lin}} \approx P \alpha \frac{\delta T}{T}
$$

Obviously, we see that in the linear regime the open-circuit thermovoltage $V^{\text {lin }}$ is directly related to the closed-circuit thermocurrent, $I^{\text {lin }}=\sigma V^{\text {lin }}$. In particular, the dependence of the conversion efficiency on the polarization $P$ and the temperature gradient are the same. This simple picture drastically changes if one goes beyond the linear-response regime, i.e., $\delta T \sim T$. We see below that the nonlinear regime is essential in order to optimize the sensitivity for thermometry applications [9].

\section{Intrinsic noise of the TE element}

We now address the zero-frequency noise performance of the $N$-FI-S junction. In this case, the main source of noise is the current noise $\left(S_{I}\right)$ generated in the TE that is described by generalizing the expression derived in Ref. [35] in the presence of a ferromagnetic tunneling barrier:

$$
S_{I}=\frac{2}{R_{T}} \int_{-\infty}^{\infty} d E\left[N_{+}+P N_{-}\right] \mathcal{M}\left(E, V, T_{N}, T_{S}\right),
$$

where

$\mathcal{M}=f_{N}\left(V, T_{N}\right)\left[1-f_{S}\left(T_{S}\right)\right]+f_{S}\left(T_{S}\right)\left[1-f_{N}\left(V, T_{N}\right)\right]$,

and the bias $V$ is given by the solution of Eq. (4). We note that the previous formula describes both thermal, i.e., $e V \ll k_{B} T_{N}, k_{B} T_{S}$, and shot noise, i.e., $k_{B} T_{N}, k_{B} T_{S} \ll e V$, and holds in the tunneling regime.

The previous expression simplifies in the linear-response regime discussed before where we can neglect any term $\mathcal{O}(\delta T)$ in Eq. (11) finding the thermal noise 


$$
S_{I}^{\operatorname{lin}}=\frac{4}{R_{T}} \int_{-\infty}^{\infty} d E\left[N_{+}+P N_{-}\right] f_{N}(T)\left[1-f_{S}(T)\right],
$$

which may be expressed as

$$
S_{I}^{\operatorname{lin}}=4 k_{B} T \sigma,
$$

where $\sigma$ is the TE electric conductance of Eq. (6). In the open-circuit configuration, it is more convenient to write the voltage-noise spectral density

$$
S_{V}^{\operatorname{lin}}=4 k_{B} T / \sigma .
$$

Below we show that in the nonlinear regime one can approximate Eqs. (14) and (15) by substituting $\sigma$ by $1 / R_{d}$, where $R_{d}$ is the TE differential resistance.

\section{TEMPERATURE-TO-VOLTAGE CONVERSION}

When the TE is in an open-circuit configuration $\left(R_{L} \rightarrow \infty\right)$, one can realize a temperature-to-voltage conversion scheme. In such case, no charge current flows through the TE,

$$
I_{\mathrm{TE}}\left(V_{0}, T_{S}, T_{N}, h_{\mathrm{exc}}, P\right)=0 .
$$

Then, a voltage $V_{0}$ develops across the TE for $\delta T \neq 0$. The value of $V_{0}$ can be obtained from the solution of Eq. (16). The results are shown in the two upper panels of Fig. 4. Specifically, Fig. 4(a) shows the dependence of $V_{0}$ on $T_{S}$ for different values of $h_{\mathrm{exc}}, P=0.9$, and $T_{N}=0.01 T_{c}$. The increase of $T_{S}$ from the minimal temperature $T_{N}$ leads first to an enhancement of $\left|V_{0}\right|$. A further increase of $T_{S}$ leads to the suppression of the superconducting energy gap and a corresponding suppression of $V_{0}$. The voltage $V_{0}$ vanishes when superconductivity is fully suppressed for $T_{S} \rightarrow T_{c}^{*}$. We note that $V_{0}$ reaches zero continuously, owing to the fact that we choose values of $h_{\mathrm{exc}}$ for which the superconducting normal-state transition is of the second order [36].

A different temperature behavior of $V_{0}$ is obtained when $S$ is kept at $T_{S}=0.01 T_{c}$ and $T_{N}$ is varied, as shown in Fig. 4(b). In particular, besides the obvious change of sign, $V_{0}$ grows monotonically by increasing $T_{N}$ until it reaches an asymptotic value. It is important to stress that the curves $V_{0}\left(T_{N}\right)$ depend strongly on the polarization $P$ of the barrier [see Fig. 4(b)]. In particular, the larger $P$, the larger is the thermovoltage $V_{0}\left(T_{N}\right)$ developed across the TE. By contrast, the $V_{0}\left(T_{S}\right)$ amplitude turns out to be almost unaffected by the value of $P$.

The different behaviors as a function of $\delta T$ allow one to reconstruct both the amplitude and direction of the thermal gradient in the TE element. This further information can be eventually exploited to reconstruct the spatial position of a heating event, thereby opening interesting possibilities to build detectorlike devices.
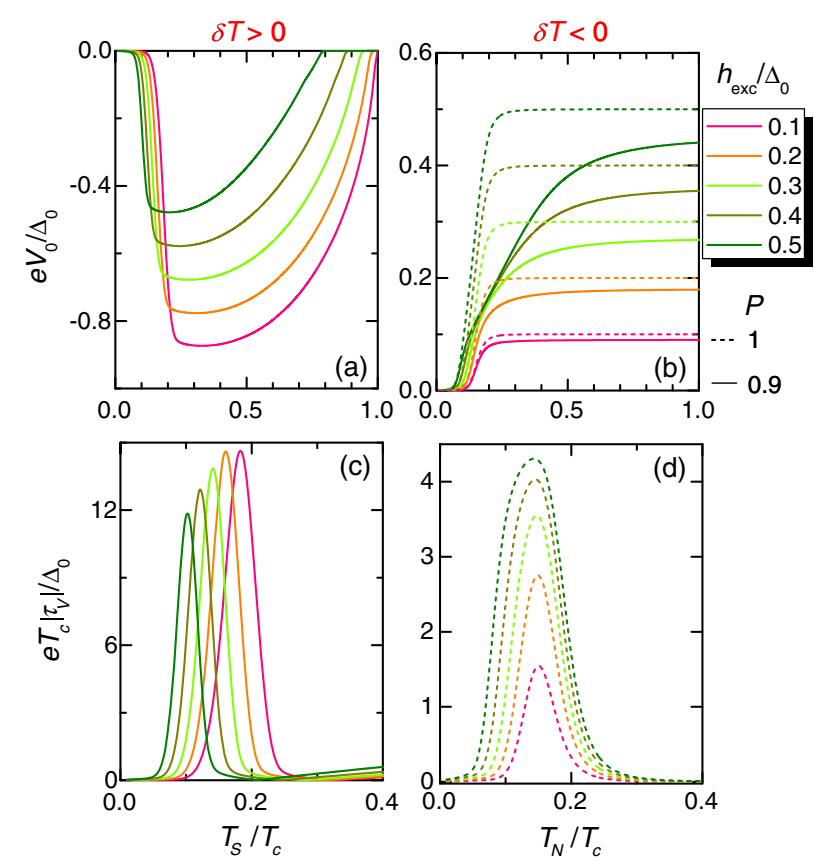

FIG. 4. Open-circuit configuration. (a) Thermovoltage $V_{0}$ vs $T_{S}$ calculated for a few values of $h_{\text {exc }}$ (see legend) at $T_{N}=0.01 T_{c}$ and $P=0.9$. (b) $V_{0}$ vs $T_{N}$ calculated for a few values of $h_{\mathrm{exc}}$ at $T_{S}=0.01 T_{c}$ and $P=0.9$ (full lines) or $P=1$ (dashed lines). (c) and (d) show the absolute value of the corresponding temperature-to-voltage transfer function $\left|\tau_{V}\right|$ calculated for the same values of $h_{\text {exc }}$.

An useful figure of merit to estimate the performance of the TE is the temperature-to-voltage transfer function, $\tau_{V}=\partial V / \partial T$. The absolute value of this quantity is shown in Fig. 4(c) for $\delta T>0$ and in Fig. 4(d) for $\delta T<0$. We normalize it to the natural unit $\Delta_{0} / e T_{c}$. In Fig. 4(d), we show the case of barrier polarization selectivity $P=1$, which corresponds to the case with a maximal possible transfer function at given $h_{\mathrm{exc}}$.

In order to show the impact of the broadening parameter, we display in Fig. 5 the same quantities as in Fig. 4 but calculated for fixed $h_{\text {exc }}=0.3 \Delta_{0}, P=0.9$, and for different values of $\Gamma$ ranging from $10^{-6} \Delta_{0}$ to $10^{-2} \Delta_{0}$ [37-39]. The overall qualitative behavior and the order of magnitude of the effect are the same for all these values. From a quantitative point of view, the temperature-to-voltage conversion turns out to be less effective the larger the value of $\Gamma$. Throughout the paper, we assume $\Gamma=10^{-4} \Delta_{0}$, which is the typical value for conventional Al-based superconducting junctions $[9,37]$.

\section{A. Noise-performance analysis for the open-circuit configuration}

We now focus our analysis on the noise performance of the temperature-to-voltage conversion with the $\mathrm{N}$-FI-S junction. We need to convert it to a voltage-noise assuming that load resistance $R_{L} \rightarrow \infty$. This means that the 

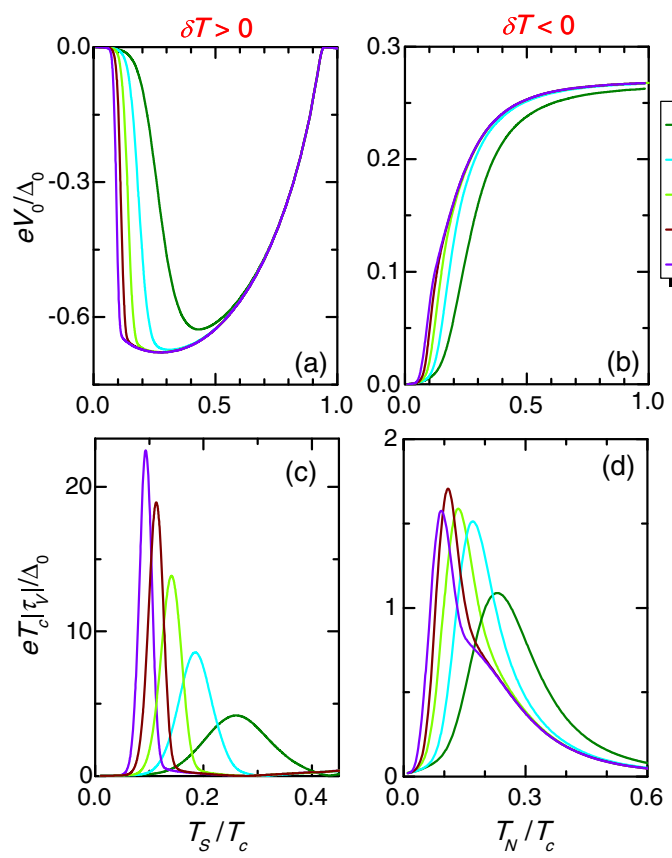

FIG. 5. Open-circuit configuration. (a) Thermovoltage $V_{0}$ vs $T_{S}$ calculated for a few values of $\Gamma$ (see legend) at $T_{N}=0.01 T_{c}$, $h_{\mathrm{exc}}=0.3 \Delta_{0}$, and $P=0.9$. (b) $V_{0}$ vs $T_{N}$ calculated for a few values of $\Gamma$ at $T_{S}=0.01 T_{c}, h_{\mathrm{exc}}=0.3 \Delta_{0}$, and $P=0.9$ (full lines). (c) and (d) show the absolute value of the corresponding temperature-to-voltage transfer function $\left|\tau_{V}\right|$ calculated for the same values of $\Gamma$.

voltage-noise spectral density $\left(S_{V}\right)$ generated across the TE is [40]

$$
S_{V}\left(V_{0}, T_{S}, T_{N}, h_{\mathrm{exc}}, P\right)=S_{I} R_{d}^{2}
$$

where the $R_{d}=\partial V_{0} / \partial I_{\mathrm{TE}}$ is the differential resistance of the TE, and the bias $V_{0}$ is given by the solution of Eq. (16).

In Figs. 6(a) and 6(b), the square root of noise spectral density $\left(\sqrt{S_{V}}\right)$ is displayed for a TE element with a barrier characterized by a realistic value of polarization $P=0.98$. This spin-filter efficiency is representative for $\mathrm{EuO}$ or EuS FI barriers [41], and we assume a superconductor with $T_{c}=3 \mathrm{~K}$ which will be implementable with ultrathin Al films [29-32].

We find that for $\delta T>0$, the minimal noise value is obtained in the nonlinear regime $T_{N} \ll T_{S} \lesssim T_{c}$ where the voltage noise can be as low as approximately $600 \mathrm{fV} \mathrm{Hz}^{-1 / 2}$ and is 2 orders of magnitude lower than the equilibrium case $\delta T / T \ll 1$, where $T=\left(T_{S}+T_{N}\right) / 2$ is the average temperature. For $\delta T<0$, the noise performance is worse, being at best a few tens of $\mathrm{pV} \mathrm{Hz}^{-1 / 2}$ for the nonlinear regime $T_{S} \ll T_{N} \sim T_{c}$.

The intrinsic temperature noise (temperature sensitivity) per unit bandwidth of the thermometer $\left(s_{T}\right)$ is related to the voltage-noise spectral density as
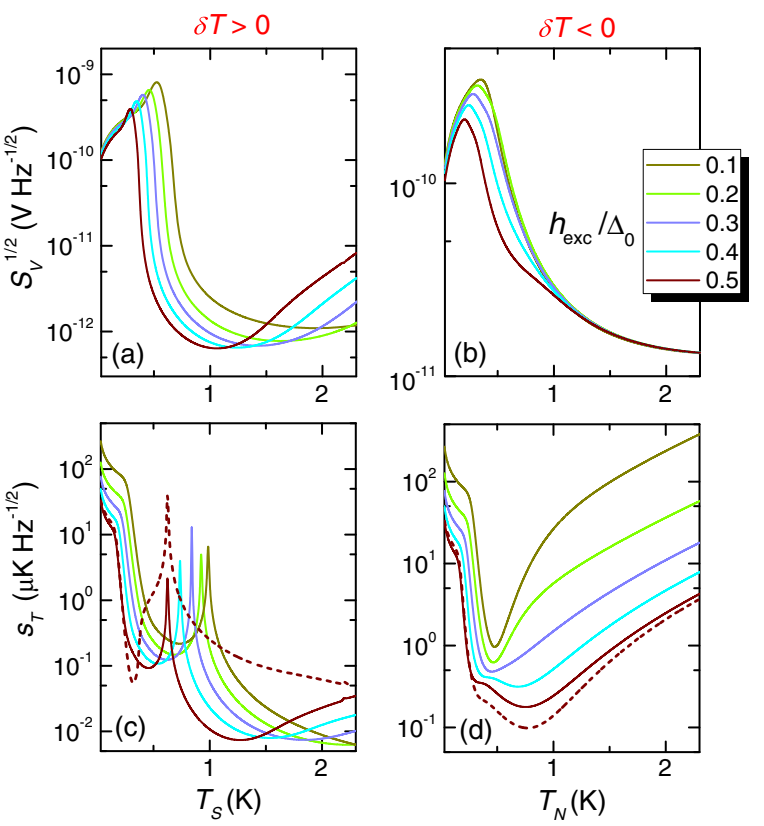

FIG. 6. Open-circuit configuration. (a) Square root of the voltage-noise spectral density $S_{V}^{1 / 2}$ vs $T_{S}$ calculated at $T_{N}=0.01 T_{c}$ for a few values of $h_{\text {exc }}$ (see legend). (b) $S_{V}^{1 / 2}$ vs $T_{N}$ calculated at $T_{S}=0.01 T_{c}$ for the same $h_{\text {exc }}$ values. (c) Temperature sensitivity $s_{T}$ vs $T_{S}$ calculated for the case of panel (a). (d) $s_{T}$ vs $T_{N}$ calculated for the case of panel (b). Dashed lines show $s_{T}$ as calculated using the approximate formula Eq. (19) for $h_{\mathrm{exc}} / \Delta_{0}=0.5$. In all these calculations, we set $P=0.98$ and $T_{c}=3 \mathrm{~K}$.

$$
s_{T}=\frac{\sqrt{S_{V}}}{\left|\tau_{V}\right|}
$$

In Figs. 6(c) and 6(d), we show the temperature noise $s_{T}$ for the open-circuit configuration for the two cases of Figs. 6(a) and 6(b). The differences between the voltage spectral density is entirely given by the transfer function which is highly nonlinear as a function of $\delta T$. We notice that in the linear regime $|\delta T| / T \ll 1$, the temperature noise given by Eq. (15) is a few tens of $\mu \mathrm{K} \mathrm{Hz}^{-1 / 2}$. The maximum temperature sensibility is obtained in the nonlinear regime, i.e., $|\delta T| / T \gg 1$, where the temperature noise can be as low as $8 \mathrm{nK} \mathrm{Hz}^{-1 / 2}$ coinciding with the minimal voltage noise. By contrast, for the case $\delta T<0$, the best noise performance is around $180 \mathrm{nK} \mathrm{Hz}^{-1 / 2}$.

It is interesting to observe the scaling behavior of noise power as a function of the junction normal-state resistance $R_{T}$. Indeed, as $S_{I} \propto 1 / R_{T}$ [see Eq. (11)], from Eq. (17) one can conclude that $S_{V} \propto R_{T}$ since $R_{d} \propto R_{T}$. At the same time, there is no scaling behavior of the transfer function, $\tau_{V}=\partial V_{0} / \partial T$. This may be easily inferred, for instance, from the expression of the thermovoltage $V_{0}$ in the linear regime, Eq. (9), since $V^{\text {lin }} \propto \alpha / \sigma$, which is the ratio of two quantities with the same scaling $1 / R_{T}$. Alternatively, one can deduce it 
from the relation between open-circuit voltage and the temperature difference, $\delta T$ Eq. (16), where $R_{T}$ enters only as an overall prefactor. We conclude that $s_{T} \propto \sqrt{R_{T}}$, which shows immediately that the reduction of TE resistance will be beneficial for increasing the sensitivity in temperature measurement.

These considerations suggest that in the nonlinear regime the differential resistance $R_{d}$ takes the role of $R_{T}$. Indeed, one can guess a way to generalize Eq. (15) to the nonlinear regime by replacing the linear conductance $\sigma$ by the differential conductance $1 / R_{d}$ such that

$$
S_{V} \approx 4 k_{B} T R_{d}
$$

where the temperature is taken as the average $T=\left(T_{S}+T_{N}\right) / 2$. The previous expression should converge to the linear result when $|\delta T| / T \ll 1$. The full numerical results of Figs. 6(c) and 6(d) demonstrate the accuracy of Eq. (19) shown as dashed lines. This shows that the noise performance is essentially characterized by the dependence of differential resistance $R_{d}$. Therefore, this approximation is extremely useful to estimate the noise performance with the knowledge of the differential resistance $R_{d}$ only.

It is important to emphasize that in a realistic measurement scheme, the temperature sensitivity of the device is limited by the amplifying chain. Indeed, in general, the voltage signal must be amplified with a low-noise preamplifier which is characterized by its intrinsic voltage noise. The preamplifier noise may degrade the total noise performance. In particular, assuming for the preamp a square-root spectral density of approximately $1 \mathrm{nV} \mathrm{Hz}^{-1 / 2}$, it is clear that it will dominate over the intrinsic voltage noise of the signal which can be smaller by a few orders of magnitude [see Figs. 6(a) and 6(b)]. The preamplifier is, therefore, the main bottleneck in the temperature detection in this configuration scheme, although it has the advantage of suppressing the noise nonlinearities over the considered temperature window. The realistic performance of this measurement scheme will be roughly $10 \mu \mathrm{K} \mathrm{Hz}^{-1 / 2}$. This limitation can be overcome by exploiting a closed-circuit configuration, as we discuss in the next section.

\section{TEMPERATURE-TO-CURRENT CONVERSION}

Hereafter, we analyze the performance of a closedcircuit configuration which corresponds to temperatureto-current conversion. In this setup, the TE current $I_{\mathrm{TE}}(V=0)=I_{\mathrm{TE}}^{0}$ is given by Eq. (3), which depends only on $T_{S}$ and $T_{N}$.

In Fig. 7(a), we show how the current depends on $T_{S}$ for different values of $h_{\text {exc }}$ keeping fixed the barrier polarization $P=0.98$ and $T_{N}=0.01 T_{c}$. The general behavior has a "shark-fin" shape, which increases in amplitude with the $h_{\text {exc }}$. After reaching a maximum at
$T_{S}^{*}$, the $I_{\mathrm{TE}}^{0}$ decreases with $T_{S}$ until the critical temperature $T_{c}^{*}$ is reached and the superconductivity is completely suppressed.

If we fix $T_{S}=0.01 T_{c}$, by changing $T_{N}$ we get for $\delta T<0$, an obvious opposite sign for the thermocurrent $I_{\mathrm{TE}}^{0}$ and an absolute value of the thermocurrent $\left|I_{\mathrm{TE}}^{0}\right|$, which monotonously increases by enhancing $|\delta T|$. It finally saturates to the maximal value

$$
I_{\mathrm{TE}, \max }^{0}=\frac{1}{2 e R_{T}} \int_{-\infty}^{\infty} d E\left[N_{+}+P N_{-}\right] \operatorname{sgn}(E),
$$

which is easily obtained from the general expression of the TE current, Eq. (3), by taking the limit $T_{S} \rightarrow 0$ and $T_{N} \rightarrow \infty$ with $V=0$. From this result, we can conclude that an arbitrary enhancement of $|\delta T|$ is not of particular benefit to increase the current signal.

In Figs. 7(c) and 7(d), we show the absolute value of the temperature-to-current transfer function $\tau_{I}=\partial I_{\mathrm{TE}}^{0} / \partial T$, respectively, for Figs. $7($ a) and $7($ b). For $\delta T>0$, the transfer function has two different behaviors depending if $T_{S}$ is smaller or larger than $T_{S}^{*}$. On the other hand, one sees that independent of the sign of $\delta T$, the transfer function is maximized in the nonlinear regime $|\delta T| \sim T$.
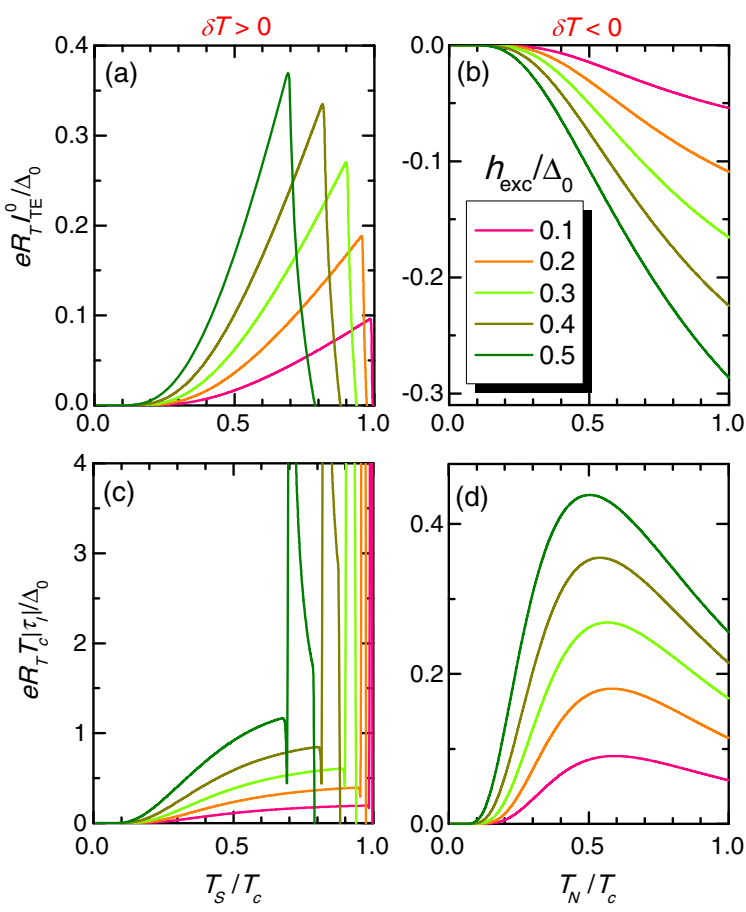

FIG. 7. Closed-circuit configuration. (a) Thermocurrent $I_{\mathrm{TE}}^{0}$ vs $T_{S}$ calculated for a few values of $h_{\mathrm{exc}}$ at $T_{N}=0.01 T_{c}$. (b) $I_{\mathrm{TA}}^{0} \mathrm{vs}$ $T_{N}$ calculated for the same values of $h_{\text {exc }}$. (c) and (d) show the absolute value of the temperature-to-current transfer function $\left|\tau_{I}\right|$ vs temperature for panels (a) and (b), respectively, calculated for the same values of $h_{\mathrm{exc}}$. In all these calculations, we set $P=0.98$. 


\section{A. Noise-performance analysis for the closed-circuit configuration}

In Figs. 8(a) and 8(b), the current noise $S_{I}$ of the closedcircuit configuration is shown as obtained from Eq. (11) with $V=0$. The current noise as a function of $\delta T$ is minimized in the linear regime obtaining approximately $15 \mathrm{fA} \mathrm{Hz}^{-1 / 2}$ and grows by increasing $|\delta T|$. The noise behavior of the closed-circuit configuration it less affected by the sign of $\delta T$ in comparison to the open-circuit one (see Sec. III A). The current noise increases with $h_{\mathrm{exc}}$ since also the average current $I_{\mathrm{TE}}^{0}$ increases in such a case [see Figs. 7(a) and 7(b)].

The intrinsic temperature noise per unit bandwidth of the thermometer $s_{T}$ in this configuration is given by

$$
s_{T}=\frac{\sqrt{S_{I}}}{\left|\tau_{I}\right|}
$$

where $\left|\tau_{I}\right|$ is the temperature-to-current transfer function discussed before. The same scaling behavior shown before for $s_{T} \propto \sqrt{R_{T}}$ still holds in this configuration, since now $S_{I} \propto 1 / R_{T}$ but $\tau_{I} \propto 1 / R_{T}$. Consequently, also for this case the minimization of $R_{T}$ would be, in general, beneficial for improving noise performance. As in the previous section,
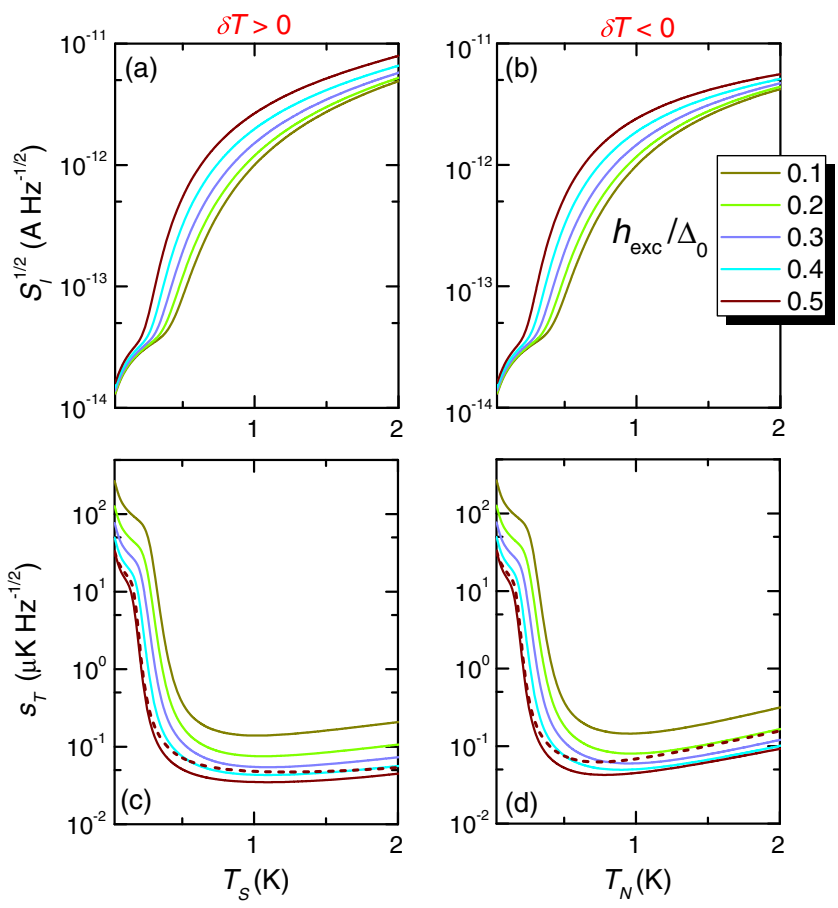

FIG. 8. Closed-circuit configuration. (a) Square root of the current noise spectral density $S_{I}^{1 / 2}$ vs $T_{S}$ for $T_{N}=0.01 T_{c}$ and for different values of $h_{\mathrm{exc}}$ (see legend). (b) $S_{I}^{1 / 2}$ vs $T_{N}$ for $T_{S}=0.01 T_{c}$ for the same values of $h_{\mathrm{exc}}$. (c) $s_{T}$ vs $T_{S}$ for the case of panel (a). (d) $s_{T}$ vs $T_{N}$ for the case of panel (b). Dashed lines show $s_{T}$ as calculated using the approximate formula Eq. (22) for $h_{\text {exc }} / \Delta_{0}=0.5$. In all these calculations, we set $P=0.98$ and $T_{c}=3 \mathrm{~K}$. one can try to generalize this argument for the nonlinear regime by replacing $R_{T}$ with $R_{d}$. Since $R_{d}$ is largely reduced in comparison to the linear regime value $1 / \sigma$, one can expect an increase of the noise in the nonlinear regime. At a first glance, this does not look plausible since the current noise is, in general, higher [see Figs. 8(a) and 8(b)]. However, our guess seems to be correct, as shown in Figs. 8(c) and 8(d), where the temperature noise is minimized for large values of $\delta T$.

The lowest intrinsic noise of approximately $35 \mathrm{nK} \mathrm{Hz}^{-1 / 2}$ is obtained in the nonlinear regime for $\delta T \approx 1 K$, when $P=0.98$ and $T_{c}=3 \mathrm{~K}$ are chosen. The main difference with the open-circuit configuration (see Fig. 6) is that in the present situation the noise depends weakly on the sign of $\delta T$ in a wide temperature region. Moreover, in contrast to the open-circuit configuration, the noise shows a rather smooth behavior.

As pointed out above, the behavior of the current noise in the nonlinear regime can be approximated by the expression

$$
S_{I} \approx \frac{4 k_{B} T}{R_{d}},
$$

where the linear conductance $\sigma$ of Eq. (14) is replaced by the inverse differential resistance, $1 / R_{d}$. In Figs. 8 (c) and $8(\mathrm{~d})$, we show (dashed lines) this approximation for the case where we expect the largest nonlinearities, i.e., for $h_{\text {exc }} / \Delta_{0}=0.5$. We can, thus, conclude that this simple formula gives a fairly accurate description of $S_{I}$ in the nonlinear regime.

In terms of overall temperature noise, the closed-circuit configuration has two advantages: First, the smooth behavior of temperature noise makes it more attractive than the open-circuit configuration. Second, while the ideal noise is better for the open-circuit configuration (see Figs. 6 and 8), one needs to evaluate the total noise of the measurement which includes the addition of the preamplifier noise. The latter noise, as we discussed in the previous section, strongly degrades the resulting noise figure. The closedcircuit configuration offers a way to overcome this limitation, as we discuss in the following.

Specifically, we propose to measure the current signal by coupling the closed circuit via a mutual inductance $M$ to a superconducting quantum-interference device (SQUID), in order to measures the flux generated by the current circulating in the thermoelectric circuit. The total temperature sensitivity, which includes now the SQUID noise, can be written as

$$
s_{T}^{\mathrm{tot}}=\frac{\sqrt{S_{\phi}^{\mathrm{tot}}}}{\left|\tau_{\phi}\right|}=\frac{\sqrt{S_{I}+\left(S_{\phi}^{\mathrm{SQUID}} / M^{2}\right)}}{\left|\tau_{I}\right|},
$$

where the temperature-to-flux transfer function is $\tau_{\phi}=M \tau_{I}$, and the TE flux spectral density $S_{\phi}^{\mathrm{TE}}=M^{2} S_{I}$ 
is added to the SQUID noise ( $S_{\phi}^{\mathrm{SQUID}}$ ) to give the total flux noise, $S_{\phi}^{\mathrm{tot}}=S_{\phi}^{\mathrm{TE}}+S_{\phi}^{\mathrm{SQUID}}$. The square root of the flux noise for a high-quality commercial SQUID can be as low as $\sqrt{S_{\phi}^{\text {SQUID }}} \sim 10^{-7} \Phi_{0} \mathrm{~Hz}^{-1 / 2}$, which is then converted into an effective circulating current noise in the thermoelectric circuit of approximately $20 \mathrm{fA} \mathrm{Hz}^{-1 / 2}$ by dividing it with a typical value for the mutual inductance $M=10^{-8} \mathrm{H}$. By looking at Figs. 8(a) and 8(b), we immediately see that the intrinsic TE current noise will, in general, dominate over the SQUID noise almost everywhere in the nonlinear regime where we can achieve the best sensitivity. Therefore, the temperature noise of this measurement scheme is limited only by the intrinsic TE noise mechanisms and can be as low as approximately $35 \mathrm{nK} \mathrm{Hz}^{-1 / 2}$ for a moderate-temperature nonlinearity (see Fig. 8).

\section{TEMPERATURE-TO-FREQUENCY CONVERSION}

We now focus on the temperature-to-frequency conversion process. This conversion is achieved with the device sketched in Fig. 1 where the thermovoltage generated across the TE is applied to a generic Josephson element which is set to operate in the dissipative regime when $I_{\mathrm{JJ}}=V / R_{\mathrm{JJ}}$ (where $R_{\mathrm{JJ}}$ is the total shunting resistance of the Josephson element). In this case, there is a timeoscillating current through the Josephson element with a frequency equal to the Josephson frequency, $\nu=|V| / \Phi_{0}$. As we discuss above, the value of $V$ depends on the temperature difference $\delta T$ across the TE, and, therefore, the frequency emitted by the Josephson junction is a measure of $\delta T$.

In order to quantify the temperature-to-frequency conversion effect, one has to determine the voltage $V$ developed for any given $\delta T$ imposed across the TE which satisfies Eq. (4) with a finite load resistance $R_{L}=R_{\mathrm{JJ}}$. This configuration is intermediate between the open-circuit and the closed-circuit setup that we discuss above. In the following, we consider the case where $R_{T} / R_{\mathrm{JJ}}=0.2$ in order to produce a detectable frequency signal between $10 \mathrm{GHz}$ and fractions of terahertz.

The frequency-to-temperature performance of this configuration is shown in Fig. 9. We again use the spin-filter efficiency $P=0.98$ and the critical temperature $T_{c}=3 \mathrm{~K}$ adopted in the previous sections. Figures 9 (a) and 9(b) show the frequency generated by the Josephson element for positive and negative $\delta T$, respectively. In the linearresponse regime, the TE thermovoltage depends only on $|\delta T|$ as can be seen from Fig. 9. The information about the sign of $\delta T$ is eventually recovered only for the nonlinear regime.

If $T_{N}$ is kept at $0.01 T_{c}$, the maximum frequency is achieved around $h_{\mathrm{exc}} \approx 0.2 \Delta_{0}$ for $T_{S} \approx 0.75 T_{c}$ and obtains
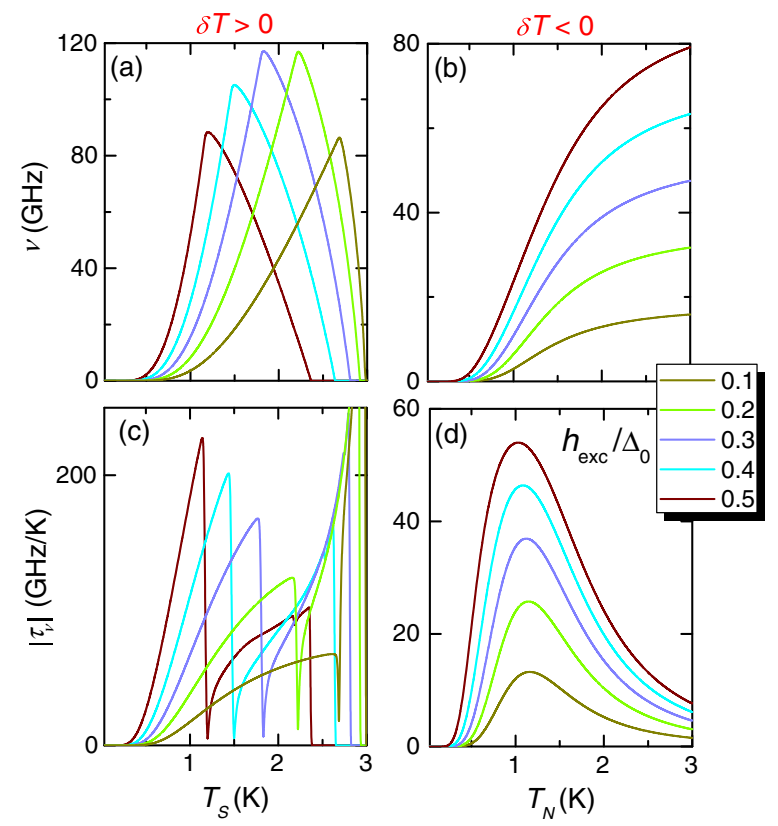

FIG. 9. Characterization of the temperature-to-frequency converter. (a) Frequency $\nu$ vs $T_{S}$ calculated for a few values of $h_{\text {exc }}$ at $T_{N}=0.01 T_{c}$. (b) $\nu$ vs $T_{N}$ at $T_{S}=0.01 T_{c}$ calculated for the same $h_{\text {exc }}$ values as in panel (a). (c) and (d) show the absolute value transfer function $\left|\tau_{\nu}\right|$ of panel (a) and (b), respectively, calculated for the same values of $h_{\text {exc }}$. In all these calculations, we set $P=0.98, R_{T} / R_{J J}=0.2$, and $T_{c}=3 \mathrm{~K}$.

values as large as approximately $120 \mathrm{GHz}$. If $T_{S}$ is kept at low temperature, $\nu$ increases monotonically by increasing both $T_{N}$ and/or $h_{\text {exc }}$ and obtains a maximum of approximately $80 \mathrm{GHz}$.

In the present setup, an important figure of merit of the structure is represented by the temperature-to-frequency transfer function $\tau_{\nu}=\partial \nu / \partial T$ plotted in absolute value in Figs. 9(c) and 9(d). In particular, $\left|\tau_{\nu}\right|$ exceeding $200 \mathrm{GHz} / \mathrm{K}$ around $T_{S} \sim 1 \mathrm{~K}$ can be achieved for $h_{\mathrm{exc}}=0.5 \Delta_{0}$ by heating $S$, while $\left|\tau_{\nu}\right|$ up to approximately $55 \mathrm{GHz} / \mathrm{K}$ can be achieved with the same values by heating $N$.

\section{A. Noise performance}

In the temperature-to-frequency conversion process, the noise is determined by the bias fluctuations generated from the current noise via the load resistance seen by the TE, i.e., the parallel between the Josephson element total resistance $R_{\mathrm{JJ}}$ and the TE resistance $R_{d}$ : $\mathcal{R}=R_{d} R_{\mathrm{JJ}} /\left(R_{d}+R_{\mathrm{JJ}}\right)$. Note that the differential resistance $R_{d}=\partial V_{0} / \partial I_{\mathrm{TE}}$ is calculated from the solutions of Eq. (4) where $R_{L}=R_{\mathrm{JJ}}$. The important quantity is represented by the frequency-noise spectral density $\left(S_{\nu}\right)$, which can be expressed as

$$
S_{\nu}=\frac{S_{I} \mathcal{R}^{2}}{\Phi_{0}^{2}}
$$



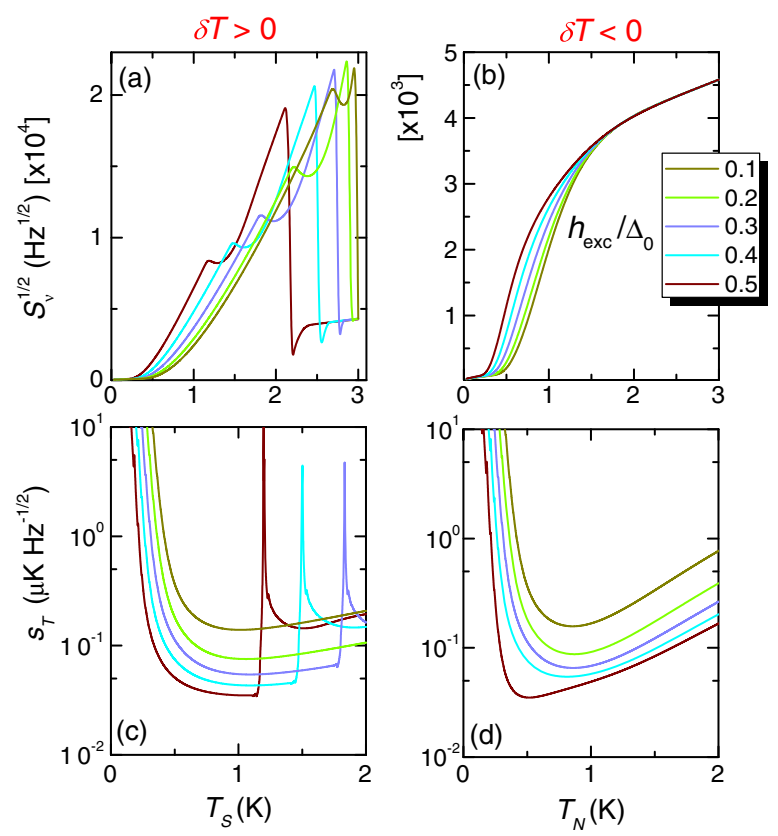

FIG. 10. Noise performance of the temperature-to-frequency converter. (a) Square root of the frequency-noise spectral density $S_{\nu}^{1 / 2}$ vs $T_{S}$ calculated at $T_{N}=0.01 T_{c}$ for a few values of $h_{\text {exc }}$. (b) $S_{\nu}^{1 / 2}$ vs $T_{N}$ calculated at $T_{S}=0.01 T_{c}$ for the same $h_{\text {exc }}$ values. (c) Temperature sensitivity $s_{T}$ vs $T_{S}$ calculated at $T_{N}=0.01 T_{c}$ for a few values of $h_{\text {exc }}$. (d) $s_{T}$ vs $T_{N}$ calculated at $T_{S}=0.01 T_{c}$ for the same $h_{\text {exc }}$ values. In all these calculations, we set $P=0.98, T_{c}=3 \mathrm{~K}, R_{T} / R_{\mathrm{JJ}}=0.2$, and $R_{T}=1 \Omega$.

Finally, the intrinsic temperature noise per unit bandwidth of the thermometer $\left(s_{T}\right)$ is related to the frequency-noise spectral density as

$$
s_{T}=\frac{\sqrt{S_{\nu}}}{\left|\tau_{\nu}\right|} .
$$

Figures 10(a) and 10(b) show the calculated square root of the frequency-noise spectral density $S_{\nu}$ for positive and negative $\delta T$, respectively, calculated for the same parameters as in Fig. 9, and for $R_{T}=1 \Omega$. In particular, for positive $\delta T$, the noise spectrum $S_{\nu}^{1 / 2}$ shows a nonmonotonic behavior with a maximum at intermediate temperatures, and suppression at higher $\delta T$. By contrast, for $\delta T<0$ the noise spectrum grows monotonically with $|\delta T|$, and it is less influenced by $h_{\text {exc }}$.

The behavior of $s_{T}$ is displayed in Figs. 10(c) and 10(d). At small $|\delta T|$, in the linear regime, the noise sensitivity is given by several tens of $\mu \mathrm{K} \mathrm{Hz}^{-1 / 2}$. By increasing $|\delta T|$, the growth of $S_{\nu}^{1 / 2}$ [Figs. 10(a) and 10(b)] is advantageously compensated by the enhancement of $\tau_{\nu}$ [see Figs. 9(c) and 9(d)]. The best noise performance is obtained when $\left|\tau_{\nu}\right|$ is quite near its maximum. The values of $s_{T} \sim$ $35 \mathrm{nK} \mathrm{Hz}^{-1 / 2}$ are obtained around $1 \mathrm{~K}$ for $h_{\mathrm{exc}}=0.5 \Delta_{0}$. After the minimum of $s_{T}$, for $\delta T>0$, we see a peak due to the divergence of $\left|\tau_{\nu}\right|^{-1}$, i.e., the vanishing of the transfer function, as shown in Fig. 9(c). Differently for $\delta T<0$, one observes a smooth increase of $s_{T}$ determined by the progressive reduction of the transfer function [see Fig. 9(d)], which is a consequence of the saturation of the frequency when $T_{N} \rightarrow \infty$. We conclude by noticing that also for this case, the best noise performance is obtained in the nonlinear regime for $|\delta T| \lesssim 1 \mathrm{~K}$ where $s_{T}$ is almost independent of the sign of $\delta T$. This behavior is essentially due again to the fact that $R_{d}$ is strongly reduced in the nonlinear regime.

In this configuration, the power of the generated frequency signal might be somewhat low. Anyway, one can deploy the standard techniques in order to increase the emission power by connecting in parallel arrays of JJs $[42,43]$. The only limiting factor in that case will be the power that the TE element can sustain and transfer to the JJs. A rough estimate shows that when $R_{T} / R_{\mathrm{JJ}}=0.2$ and $R_{T}=1 \Omega$, the TE can produce a power of the order of approximately $100 \mathrm{pW}-10 \mathrm{nW}$ which will be high enough to make the (10-100)-GHz signal generated by the Josephson junction detectable.

\section{SUMMARY}

In summary, we theoretically investigate a thermoelectric structure based on a $N$-FI-S junction. We fully characterize the thermoelectrical properties of the TE both in the linear and nonlinear regimes. We assume different measurement configurations as determined by the load resistance value. In particular, we show that by exploiting realistic materials such as $\mathrm{EuS}$ or $\mathrm{EuO}$ (providing polarization $P$ up to approximately $98 \%$ ) in combination with superconducting $\mathrm{Al}$ thin films, the device provides remarkable temperaturenoise performance. We find that in the open-circuit configuration, where the temperature signal is returned via the Seebeck thermovoltage, the lowest achievable intrinsic noise of approximately $10 \mathrm{nK} \mathrm{Hz}^{-1 / 2}$ is limited by the amplifying chain. On the other side, we find that in the closed-circuit configuration, where the temperature information is encoded in the Peltier thermocurrent, one can detect the signal via a low-noise flux measurement of an inductively coupled SQUID. In such case, the temperaturenoise performance is mainly determined by intrinsic noise mechanisms, with the best value of approximately $35 \mathrm{nK} \mathrm{Hz}^{-1 / 2}$ is achievable with state-of-the-art SQUID technology. Interestingly, we identify in the differential resistance $R_{d}$ of the TE one of the main factors that determines the intrinsic noise performance of the system. This finding explains why the best noise performance is obtained in the nonlinear temperature regime, since for that regime $R_{d}$ is strongly suppressed. This behavior is a nontrivial consequence of the strong nonlinearities peculiar of the $N$-FI- $S$ junction.

We finally discuss a temperature-to-frequency converter where the obtained thermovoltage is converted through a dissipative Josephson junction into a high-frequency signal 
in the frequency window spanning from a few gigahertz up to approximately $10^{11} \mathrm{~Hz}$. In particular, we show that the device allows for the generation of Josephson radiation at a frequency that depends on both the amplitude and sign of the temperature difference across the $N$-FI- $S$ junction, therefore, opening the route for high-frequency detection associated to high-temperature sensitivity. Frequencies up to approximately $120 \mathrm{GHz}$ and large transfer functions (i.e., up to $200 \mathrm{GHz} / \mathrm{K}$ ) around approximately $1-2 \mathrm{~K}$ can be obtained in a structure implementable with the abovementioned prototype FIs. In this configuration, the device is capable of providing intrinsic temperature noise down to approximately $35 \mathrm{nK} \mathrm{Hz}^{-1 / 2}$ around $1 \mathrm{~K}$ for a sufficiently large $h_{\text {exc }}$. The proposed superconducting hybrid structure has the potential for the realization of effective on-demand on-chip temperature-to-frequency converters as well as ultrasensitive electron thermometers or radiation sensors easily integrable with current superconducting electronics.

\section{ACKNOWLEDGMENTS}

We acknowledge J.S. Moodera and J. W. A. Robinson for fruitful comments. F. G. acknowledges the European Research Council (ERC) under the European Union's Seventh Framework Program No. FP7/2007-2013 ERC Grant No. 615187-COMANCHE for funding. F. G. and P.S. acknowledge the MIUR-FIRB2013 Project Coca (Grant No. RBFR1379UX) for partial financial support. P.S. has received funding from the European Union Program No. FP7/2007-2013 under REA Grant No. 630925 COHEAT. A. B. thanks for the support of the MIUR-FIRB2012 Project HybridNanoDev (Grant No. RBFR1236VV). The work of F.S.B is supported by the Spanish Ministerio de Economa y Competitividad through the Project No. FIS2014-55987-P and Grupos Consolidados UPV/EHU del Gobierno Vasco (Grant No. IT-756-13).

[1] F. Giazotto and F. S. Bergeret, Phase-tunable colossal magnetothermal resistance in ferromagnetic Josephson valves, Appl. Phys. Lett. 102, 132603 (2013).

[2] F. S. Bergeret and F. Giazotto, Phase-dependent heat transport through magnetic Josephson tunnel junctions, Phys. Rev. B 88, 014515 (2013).

[3] P. Machon, M. Eschrig, and W. Belzig, Nonlocal Thermoelectric Effects and Nonlocal Onsager Relations in a ThreeTerminal Proximity-Coupled Superconductor-Ferromagnet Device, Phys. Rev. Lett. 110, 047002 (2013).

[4] A. Ozaeta, P. Virtanen, F. Bergeret, and T. T. Heikkilä, Predicted Very Large Thermoelectric Effect in FerromagnetSuperconductor Junctions in the Presence of a SpinSplitting Magnetic Field, Phys. Rev. Lett. 112, 057001 (2014).
[5] P. Machon, M. Eschrig, and W. Belzig, Giant thermoelectric effects in a proximity-coupled superconductor-ferromagnet device, New J. Phys. 16, 073002 (2014).

[6] The thermoelectric effect predicted in Refs. [3-5] has been confirmed in a recent experiment: S. Kolenda, M. J. Wolf, and D. Beckmann, Observation of thermoelectric currents in high-field superconductor-ferromagnet tunnel junctions, arXiv: 1509.05568.

[7] F. Giazotto, J. W. A. Robinson, J. S. Moodera, and F. S. Bergeret, Proposal for a phase-coherent thermoelectric transistor, Appl. Phys. Lett. 105, 062602 (2014).

[8] F. Giazotto, T. T. Heikkilä, and F. S. Bergeret, Very Large Thermophase in Ferromagnetic Josephson Junctions, Phys. Rev. Lett. 114, 067001 (2015).

[9] F. Giazotto, T. T. Heikkilä, A. Luukanen, A. M. Savin, and J. P. Pekola, Opportunities for mesoscopics in thermometry and refrigeration: Physics and applications, Rev. Mod. Phys. 78, 217 (2006).

[10] S. Kawabata, A. Ozaeta, A. S. Vasenko, F. W. J. Hekking, and F.S. Bergeret, Efficient electron refrigeration using superconductor/spin-filter devices, Appl. Phys. Lett. 103, 032602 (2013).

[11] J. Linder and J. W. A. Robinson, Superconducting spintronics, Nat. Phys. 11, 307 (2015).

[12] D. R. Schmidt, C. S. Yung, and A. N. Cleland, Nanoscale radio-frequency thermometry, Appl. Phys. Lett. 83, 1002 (2003).

[13] S. Gasparinetti, M. J. Martínez-Pérez, S. De Franceschi, J. P. Pekola, and F. Giazotto, Nongalvanic thermometry for ultracold two-dimensional electron domains, Appl. Phys. Lett. 100, 253502 (2012).

[14] P. Torresani, M. J. Martínez-Pérez, S. Gasparinetti, J. Renard, G. Biasiol, L. Sorba, F. Giazotto, and S. De Franceschi, Nongalvanic primary thermometry of a two-dimensional electron gas, Phys. Rev. B 88, 245304 (2013).

[15] T. Faivre, D. Golubev, and J. P. Pekola, Josephson junction based thermometer and its application in bolometry, J. Appl. Phys. 116, 094302 (2014).

[16] S. Gasparinetti, K. L. Viisanen, O.-P. Saira, T. Faivre, M. Arzeo, M. Meschke, and J. P. Pekola, Fast Electron Thermometry for Ultrasensitive Calorimetric Detection, Phys. Rev. Applied 3, 014007 (2015).

[17] F. Giazotto, T. T. Heikkilä, G. Pepe, P. Helisto, A. Luukanen, and J.P. Pekola, Ultrasensitive proximity Josephson sensor with kinetic inductance readout, Appl. Phys. Lett. 92, 162507 (2008).

[18] J. Govenius, R. E. Lake, K. Y. Tan, V. Pietilä, J. K. Julin, I. J. Maasilta, P. Virtanen, and M. Möttönen, Microwave nanobolometer based on proximity Josephson junctions, Phys. Rev. B 90, 064505 (2014).

[19] T. Faivre, D. S. Golubev, and J. P. Pekola, Andreev current for low temperature thermometer, Appl. Phys. Lett. 106, 182602 (2015).

[20] A. Barone and G. Paterno, Physics and Applications of the Josephson Effect (Wiley-Interscience, New York, 1982).

[21] T. Tokuyasu, J. A. Sauls, and D. Rainer, Proximity effect of a ferromagnetic insulator in contact with a superconductor, Phys. Rev. B 38, 8823 (1988).

[22] M. Tinkham, Introduction to Superconductivity, 2nd ed. (McGraw-Hill, New York, 1996). 
[23] R. C. Dynes, J. P. Garno, G. B. Hertel, and T. P. Orlando, Tunneling Study of Superconductivity near the MetalInsulator Transition, Phys. Rev. Lett. 53, 2437 (1984).

[24] M. Silaev, P. Virtanen, T. T. Heikkilä, and F. S. Bergeret, Spin Hanle effect in mesoscopic superconductors, Phys. Rev. B 91, 024506 (2015).

[25] J. S. Moodera, T. S. Santos, and T. Nagahama, The phenomena of spin-filter tunnelling, J. Phys. Condens. Matter 19, 165202 (2007).

[26] P. G. de Gennes, Coupling between ferromagnets through a superconducting layer, Phys. Lett. 23, 10 (1966).

[27] R. Meservey and P. M. Tedrow, Spin-polarized electron tunneling, Phys. Rep. 238, 173 (1994).

[28] X. Hao, J. S. Moodera, and R. Meservey, Spin-filter effect of ferromagnetic europium sulfide tunnel barriers, Phys. Rev. B 42, 8235 (1990).

[29] B. Li, G.-X. Miao, and J. S. Moodera, Observation of tunnel magnetoresistance in a superconducting junction with Zeeman-split energy bands, Phys. Rev. B 88, 161105(R) (2013).

[30] B. Li, N. Roschewsky, B. A. Assaf, M. Eich, M. EpsteinMartin, D. Heiman, M. Münzenberg, and J. S. Moodera, Superconducting Spin Switch with Infinite Magnetoresistance Induced by an Internal Exchange Field, Phys. Rev. Lett. 110, 097001 (2013).

[31] Y. M. Xiong, S. Stadler, P. W. Adams, and G. Catelani, Spin-Resolved Tunneling Studies of the Exchange Field in EuS/Al Bilayers, Phys. Rev. Lett. 106, 247001 (2011).

[32] T. J. Liu, J.C. Prestigiacomo, and P.W. Adams, SpinResolved Tunneling Studies of the Exchange Field in EuS/Al Bilayers, Phys. Rev. Lett. 111, 027207 (2013).

[33] M. J. Wolf, C. Sürgers, G. Fischer, and D. Beckmann, Spinpolarized quasiparticle transport in exchange-split superconducting aluminum on europium sulfide, Phys. Rev. B 90, 144509 (2014).

[34] While thermalization of the $N$ layer is, in general, a simple issue due to the finite electron-phonon coupling existing in metals, in a superconducting layer the situation is more subtle due to the exponentially small electron-phonon interaction at low temperature. To this end, quasiparticle traps, i.e., normal-metal fingers tunnel coupled to the $S$ layer [9] are often used to provide effective thermalization of a superconductor at low temperature by removing hot quasiparticles originating in the nearby-connected overheated $N$ layer.

[35] D. Golubev and L. Kuzmin, Nonequilibrium theory of a hot-electron bolometer with normal metal-insulatorsuperconductor tunnel junction, J. Appl. Phys. 89, 6464 (2001).

[36] D. Saint-James, D. Sarma, and E. J. Thomas, Type II Superconductivity (Pergamon, New York, 1969); A. I. Buzdin, Proximity effects in superconductor-ferromagnet heterostructures, Rev. Mod. Phys. 77, 935 (2005).

[37] J. P. Pekola, T. T. Heikkilä, A. M. Savin, J. T. Flyktman, F. Giazotto, and F. W. J. Hekking, Limitations in Cooling Electrons Using Normal-Metal-Superconductor Tunnel Junctions, Phys. Rev. Lett. 92, 056804 (2004).

[38] J. P. Pekola, V. F. Maisi, S. Kafanov, N. Chekurov, A. Kemppinen, Yu. A. Paskin, O.-P. Saira, M. Möttönen, and J. S. Tsai, Environment-Assisted Tunneling as an Origin of the Dynes Density of States, Phys. Rev. Lett. 105, 026803 (2010).

[39] O.-P. Saira, A. Kemppinen, V. F. Maisi, and J. P. Pekola, Vanishing quasiparticle density in a hybrid $\mathrm{Al} / \mathrm{Cu} / \mathrm{Al}$ single-electron transistor, Phys. Rev. B 85, 012504 (2012).

[40] Sh. Kogan, Electronic Noise and Fluctuations in Solids (Cambridge University Press, Cambridge, England, 1996).

[41] T. S. Santos, J. S. Moodera, K. V. Raman, E. Negusse, J. Holroyd, J. Dvorak, M. Liberati, Y. U. Idzerda, and E. Arenholz, Determining Exchange Splitting in a Magnetic Semiconductor by Spin-Filter Tunneling, Phys. Rev. Lett. 101, 147201 (2008).

[42] M. J. Wengler, B. Guan, and E. K. Track, in Fifth International Symposium on Space Terahertz Technology, (IEEE, New York, 1994), p. 226.

[43] P. Barbara, A. B. Cawthorne, S. V. Shitov, and C. J. Lobb, Stimulated Emission and Amplification in Josephson Junction Arrays, Phys. Rev. Lett. 82,1963 (1999). 